Check for updates

Cite this: RSC Adv., 2019, 9, 34872

Received 7th June 2019

Accepted 24th October 2019

DOI: $10.1039 / c 9 r a 04280 f$

rsc.li/rsc-advances

\section{Efficient immobilization of ionic corrosion products by a silica-hydroxyapatite composite via a cold sintering route}

\author{
Sajid lqbal, $\uparrow^{\mathrm{ab}}$ Muhmood ul Hassan, $\uparrow^{\mathrm{a}}$ Ho Jin Ryu*a and Jong-Il Yun (D) *a
}

\begin{abstract}
We have successfully demonstrated a new method of radioactive waste immobilization by hosting a wastebearing form in another waste matrix. A cold sintering route was used to consolidate a silica-incorporated hydroxyapatite (Si-HAp) composite at $200^{\circ} \mathrm{C}$ by applying a uniaxial pressure of $500 \mathrm{MPa}$ for a short holding time of $10 \mathrm{~min}$. The higher relative sintered density of up to $98.0 \pm 1.3 \%$ was achieved by $25 \mathrm{wt} \% \mathrm{Si}$ loaded HAp. Results from high resolution $\mathrm{X}$-ray diffraction, micro-hardness, and high resolution scanning electron microscopy confirmed the densification with good mechanical strength (micro-hardness $=2.9 \pm 0.3 \mathrm{GPa}$ ). For practical applications, two kinds of wastes (25 wt\% ionic corrosion product-sorbed EDTA functionalized mesoporous silica and 75 wt\% ionic corrosion product-sorbed HAp) were mixed, consolidated and tested. The chemical stability of the solidified composite matrix was positively assessed for low leaching rates of 5.9 $\times 10^{-9}$ to $1.2 \times 10^{-5} \mathrm{~g}$ per $\mathrm{m}^{2}$ per day using a standard product consistency test. The consolidated composite can bear compressive stress up to $358 \mathrm{MPa}$, which is orders of magnitude higher than the waste acceptance criteria of $3.5 \mathrm{MPa}$. The low process temperature can make this sintering process very powerful for the immobilization of radionuclides with volatility and low boiling point. Such a low temperature solidified matrix hosting various wastes may be a promising path for waste management because of its simplicity, reliability, scalability, cost effectiveness and environmental friendliness.
\end{abstract}

\section{Introduction}

The effective management of radioactive waste has been discussed worldwide as a crucial issue for many years. The smart use of a variety of natural and anthropogenic wastes to capture and immobilize radioactive wastes could be an innovative technology to solve the gigantic environmental problems in an efficient and effective manner. ${ }^{1-3}$

The nuclear power industry has grown strongly in the past 50 years to meet global energy needs. According to the report from International Atomic Energy Agency (IAEA) 2018, about 448 nuclear power reactors with a total installed capacity of 391.721 GWe are in operation worldwide. The spent ion exchange resin, which is produced from nuclear power facilities installed worldwide, is more than $3500 \mathrm{~m}^{3}$ per year. ${ }^{1,45}$ The future of radioactive waste (RW) generated as a result of nuclear reactor operations has not been properly and effectively addressed. RW is generated in various ways, such as mining and fuel processing activities, spent fuel assemblies, ion exchange resins (IER),${ }^{6,7}$ radioisotope production laboratories, ${ }^{8}$ and other

${ }^{a}$ Department of Nuclear and Quantum Engineering, KAIST, Daejeon 34141, Republic of Korea. E-mail: hojinryu@kaist.ac.kr; jiyun@kaist.ac.kr; Fax: +82-42-350-3810 ${ }^{b}$ Chemistry Division, Pakistan Institute of Nuclear Science and Technology, P.O. Nilore, Islamabad, Pakistan

$\dagger$ Both authors have contributed equally to this work. sources (membrane process, evaporator, and filters, etc.). ${ }^{9} \mathrm{~A}$ large volume of radioactive sludge and solutions is produced by various activities related to the decontamination of laundry and working area.

In addition, the decommissioning of nuclear power plants also adds a significant amount of low and intermediate radioactive wastes. The spent ion exchange resin is one of the most secondary waste sources due to its tedious pre-treatments, conditionings and immobilization procedures. Moreover, the nuclear power plants and nuclear accidents such as the Fukushima Daiichi ${ }^{10}$ and Chernobyl nuclear power plants have released an enormous amount of contaminated water $\left({ }^{90} \mathrm{Sr}\right.$ and ${ }^{137} \mathrm{Cs}$, etc.) that needs immediate and effective treatment and management. ${ }^{11-13}$ There are many solidification methods to solidify low and intermediate level radioactive wastes such as cementation, bituminization, vitrification, polymer and geopolymer encapsulation, incineration, thermal organic reduction process $e t c .^{9,14-16}$ Ordinary Portland cement (OPC), bitumen (asphalt) and vitrification techniques are widely used to incorporate the spent IER wastes. A conventional solidification route for spent ion exchange resins by being hosted in ordinary Portland cement is outlined in Fig. 1. It is apparent that the conventional solidification flowsheet involves a variety of activities, such as dewatering, watering, drying, mixing of cement and additives, and solidification. Each step during waste conditioning contributes to increase the secondary waste 


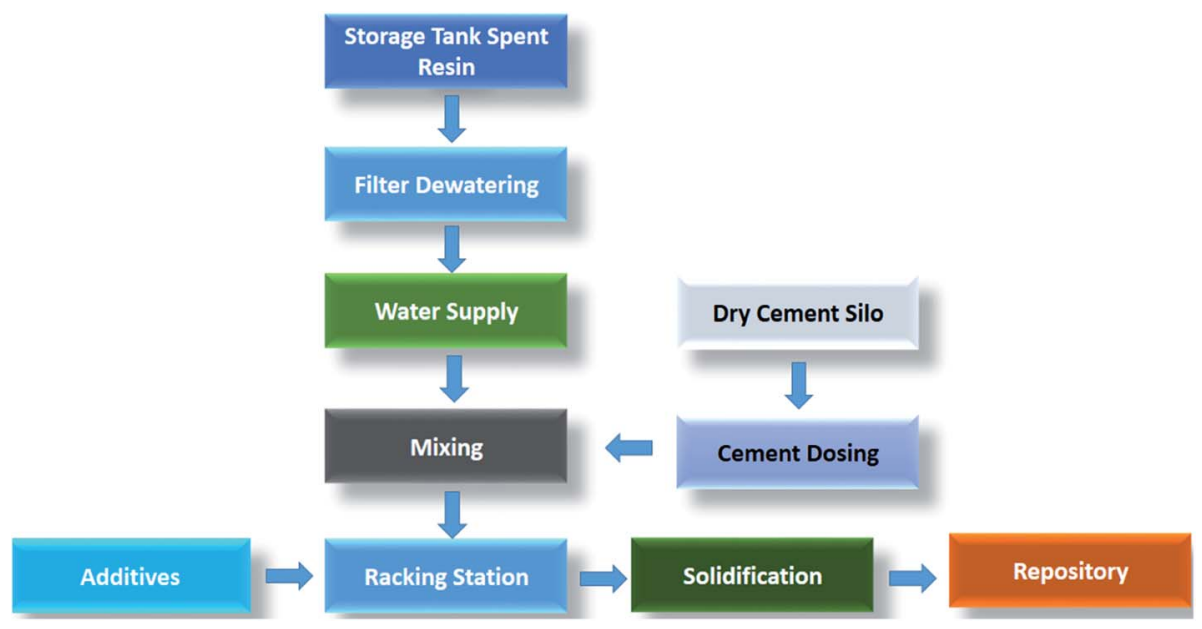

Fig. 1 Flowsheet for the conventional solidification of ion exchange resin with ordinary Portland cement.?

volume and extend the process. Therefore, it is imperative to explore a novel route for efficient and rapid waste disposal without adding binders and additives.

All available immobilization techniques have certain advantages and disadvantages associated with them. For instance, OPC has good compressive strength (15-64 MPa) and thermal stability $\left(\sim 1000^{\circ} \mathrm{C}\right)$. OPC has been readily available and widely used over the last 40 years. On the other hand, moderate leaching resistance $\left(10^{-5} \mathrm{~g}\right.$ per $\mathrm{m}^{2}$ per day), relatively less waste loading (10-25 wt \%), the requirement for binding additives, and humid atmosphere swelling are the problems of the OPC matrix. Bitumen matrix has good leaching resistance $\left(10^{-6}\right.$ to $10^{-7} \mathrm{~g}$ per $\mathrm{m}^{2}$ per day) and waste loading (25-50 wt\%) compared to OPC with low thermal stability (up to $120^{\circ} \mathrm{C}$ ); however, potential fire risk, restricting its applications in the US and many other countries. The vitrification process has fairly good waste loading ( $50 \mathrm{wt} \%$ ), the tendency to chemically bind metal ions into its amorphous network, showing excellent leaching resistance $\left(10^{-8}\right.$ to $10^{-9} \mathrm{~g}$ per $\mathrm{m}^{2}$ per day) and lowest swelling. Drawbacks of this method, however, are a high processing temperature $\left(>1200{ }^{\circ} \mathrm{C}\right)$ and an exceptional cost. Therefore, novel, efficient, cost-effective and durable materials are required to separate radionuclides and directly immobilize the immense nuclear waste that accumulates in on-site storage facilities. ${ }^{2,17}$

Here, we present a simplified, single-step radioactive waste management route for the removal of radioactive contaminants by ethylene diamine tetra acetic acid functionalized mesoporous silica (EDTA-Si) and calcium hydroxyapatite (HAp) and sintered to form composite via cold sintering for immobilization and storage. The unique property of the proposed matrix is its ability to capture radionuclides and transform itself into a mechanically and chemically durable solid composite matrix without additives. Both constituents of the composite (EDTA-Si and HAp) are readily available and possess ion exchange properties., ${ }^{3,18-21}$ HAp is the most important bioactive material with many natural sources in the form of minerals, phosphate-based rocks, and animal and fish bones. ${ }^{22}$ It can easily be synthesized by co-precipitation, sonochemical and hydrothermal methods and is commercially available as well. ${ }^{23} \mathrm{HAp}$ is widely investigated for its ion exchangeability and its ability to remove a number of ionic species from their aqueous solutions. HAp was also studied and reported for its crystal structure flexibilities to incorporate and hold a number of cationic and anionic species. ${ }^{3,24}$ Silica has been used as a thickening agent to reinforce and improve the mechanical properties of nanomaterials. ${ }^{25}$ Silica-reinforced calcium hydroxyapatite has been synthesized for dentistry and biomedical applications. ${ }^{26}$ Silicaincorporated HAp nanoparticles may increase the mechanical strength and chemical stability of nanocomposites due to micro-encapsulation phenomena. In addition, the functionalized silica nanoparticles bear excellent sorption capacities for a number of metal ions $(\mathrm{Co}, \mathrm{Cu}, \mathrm{Ni}, \mathrm{Mn}, \mathrm{Cr}, \mathrm{Zn}, \mathrm{Fe}, \mathrm{Cs}, \mathrm{Sr}){ }^{21,27}$ Therefore, it may also be possible to consolidate spent silicabased sorbents with spent HAp-based sorbents by mixing them at an optimized weight ratio and converting them into a solid monolith for final waste storage applications. In addition, the use of a spent sorbent (having no significant commercial value) to stabilize/encapsulate another waste at low temperature may have an economic advantage over other conventional solidification techniques.

Many researchers have performed high-temperature sintering $\left(>1000{ }^{\circ} \mathrm{C}\right)$ of silica-substituted HAp to enhance the mechanical properties of composites for medical applications. ${ }^{28-31}$ The immobilization of radioactive wastes by HAp has also been suggested elsewhere. ${ }^{3,32}$ Nevertheless, the sorption, solid-state mixing, and solidification properties of two spent wastes (metal ion captured silica and HAp) are the least investigated. Additionally, high-temperature sintering has certain disadvantages, such as the volatilization of sorbed volatile radionuclides, the dehydroxylation of hydroxyapatite to form unwanted apatite phases ( $\beta$-TCP), and the high processing cost. Further, the radionuclides captured by organic group functionalized sorbents can be released at high-temperature $(>200$ $\left.{ }^{\circ} \mathrm{C}\right)$ due to the degradation of the organic matrix. ${ }^{1}$ Recently, Hassan et al. reported the cold sintering of pure HAp, and cobalt 
sorbed HAp with good density, mechanical and chemical properties. ${ }^{33}$

Cold sintering is a densification process of a board set of inorganic powders at extremely low temperatures up to $\sim 200^{\circ} \mathrm{C}$ using a uniaxial pressing. ${ }^{34,35}$ The initial compaction is achieved by rearrangement of solid particles under mechanical force of the applied pressure. Thereafter, as a result of the combined effect of pressure and temperature, atoms, ions, molecules, and clusters diffuse at the interfaces to reduce the surface free energy and porosity, densely packing the particles together to achieve the maximum density.

The aim of this study is to explore new avenues for radioactive waste disposal by hosting one waste into another to form a low temperature processed waste matrix. There can be certain disadvantages if we immobilize individual sorbents by following conventional methods: such as sintering problems of ionic corrosion products (ICPs) captured EDTA-Si at low temperature, low metal loading capacity of HAp and requirement of the binding matrix (cement, bitumen, glass) to consolidate each individual sorbents. The strategy to incorporate and immobilize spent sorbents by themselves containing ICPs may be promising to enhance the overall waste loadings and reduce the secondary waste volume. Therefore, we designed and investigated the new composite consisting of a combination of spent organic and inorganic type sorbents. Standard mechanical and durability tests were performed to ensure the waste acceptance criteria for the immobilized waste matrix.

\section{Experimental}

\subsection{Chemical procedures}

Hydroxyapatite powder was synthesized by a co-precipitation method with a little modification. ${ }^{36}$ HAp is a well-known sorbent for trapping monovalent and divalent metal ions from aqueous media. ${ }^{3,37,38}$ A detailed sorption study on the optimization of the HAp powder dose to remove a maximum amount of metal ions from a mixture may be found in our previous study. ${ }^{3}$ Briefly, $40 \mathrm{~g} \mathrm{~L}^{-1}$ HAp powder was found sufficient to remove more than $95 \%$ of the metal ions $(\mathrm{Co}, \mathrm{Cr}, \mathrm{Cu}, \mathrm{Fe}, \mathrm{Mn}, \mathrm{Ni}$, and $\mathrm{Zn}$ ) with a total concentration of $7 \mathrm{mM}$ at $\mathrm{pH}$ 6. In another study, EDTA-Si was synthesized and applied to capture $\mathrm{Co}, \mathrm{Cu}$ and $\mathrm{Ni}$ ions from aqueous solution. ${ }^{21}$ Here, we applied the synthesized EDTA-Si powder ranging from 3 to $9 \mathrm{~g} \mathrm{~L}^{-1}$ to optimize the sorbent dose. More than $95 \%$ of the dissolved metal ions were removed as the sorbent dose increased to $9 \mathrm{~g} \mathrm{~L}^{-1}$ at $\mathrm{pH}$ 4. The optimized solid-to-liquid ratios for $\operatorname{HAp}\left(40 \mathrm{~g} \mathrm{~L}^{-1}\right)$ and EDTA-Si $\left(9 \mathrm{~g} \mathrm{~L}^{-1}\right)$ were used for further experiments.

\subsection{Sintering methodology for pure and ICPs sorbed Si-HAp powder}

We used the synthesized HAp powder as a host matrix for silicabased sorbents. For this purpose, commercial silica nanoparticle (Sigma Aldrich) and synthesized pure HAp powder were initially used to optimize silica loading into the HAp matrix. Fig. 2 shows the schematic diagram of mixing, densifying and sintering of silica-HAp (Si-HAp). Five samples in triplicate were prepared to take $0,1,10,20$ and $25 \mathrm{wt} \%$ of silica powder with HAp. Uniform mixing of nano-powders was performed with a 3D mixer at $30 \mathrm{rpm}$ for $12 \mathrm{~h}$ using stainless steel balls by mixing the powders with a small amount of ethanol. After mixing, ethanol was evaporated at room temperature, followed by drying the mixed powder under vacuum at $110^{\circ} \mathrm{C}$ for $12 \mathrm{~h}$. To make sintered pellets, $1.10 \mathrm{~g}$ of powder was put into a specially designed $13 \mathrm{~mm}$ stainless steel mold. The mold was wrapped with a heating band and then placed in a Carver press machine at a uniaxial pressure of $500 \mathrm{MPa}$. The ceramic heating band obtained from Samsung Electronics Co. was used to provide a constant sintering temperature of $200{ }^{\circ} \mathrm{C}$ for $10 \mathrm{~min}$. Thereafter, the mold was allowed to cool and the pressure was slowly released before the sample ejection from the mold at room temperature. Optimized cold sintering parameters (applied pressure, sintering temperature, holding time) were applied for sintering of Si-HAp-composite with silica from 1 to $25 \mathrm{wt} \%{ }^{39} \mathrm{~A}$ $25 \mathrm{wt} \%$ loading of silica powder to HAp was assumed to be the optimum loading among studied conditions because the sintered pellets start breaking during cooling and ejection from the mold at Si loading $\geq 30 \mathrm{wt} \%$. Furthermore, it was not possible to measure the physical and mechanical properties of broken pellets.

After optimizing the pure Si loading into pure HAp matrix, the cold sintering of ICPs sorbed EDTA-Si (ICPs-Si) and ICPssorbed HAp composite ( $25 \mathrm{wt} \%$ ICPs-Si and $75 \mathrm{wt} \%$ ICPs-HAp) was performed under identical sintering conditions as shown in Fig. 2.

\subsection{Characterization}

High-resolution XRD patterns were measured with SmartLab, RIGAKU, $2 \theta$ scan range from 20 to $60^{\circ}$, the step size of 0.02 at 45 $\mathrm{kV}$ and $200 \mathrm{~mA}$ at a scan speed of $4^{\circ} \mathrm{min}^{-1}$ with a $\mathrm{Cu} \mathrm{K}_{\alpha}$ incident radiation source of wavelength $(\lambda=1.54056 \AA)$ in reflection geometry. The International Center for Diffraction Data Powder Diffraction Files (HAp, ICDD-PDF) 09-0432 were used to identify the crystalline phases.

Attenuated total reflectance-Fourier transform infrared (ATR-FTIR) spectroscopy (Nicolet is50, Thermo Fisher) was used in the scan range of 4000 to $400 \mathrm{~cm}^{-1}$ by a built-in DLaTGS ATR detector with a resolution of $4 \mathrm{~cm}^{-1}$. Duplicate measurements were made for each sample using two specimens per sample.

Scanning electron microscopy (SEM) (SU5000 Hitachi) was used to examine the morphology and grain growth. The elemental mapping of the sintered samples was carried out with an Octane Elite EDX detector installed onto an SU5000 SEM device (Hitachi). Prior to SEM analysis, the sintered pellets were broken and mounted on the individual substrate holder using carbon adhesive tape coated with an Osmium Plasma Coater before sputtering.

The hardness of the sintered pellets was measured using a Vickers microindentor (Model: 402MVD, Wolpert Wilson Instruments) under an applied load of $200 \mathrm{gf}$ for $10 \mathrm{~s}$. The upper and lower surfaces of the sintered pellets were polished to $1 \mu \mathrm{m}$ before the hardness measurements. Ten indents were made in 


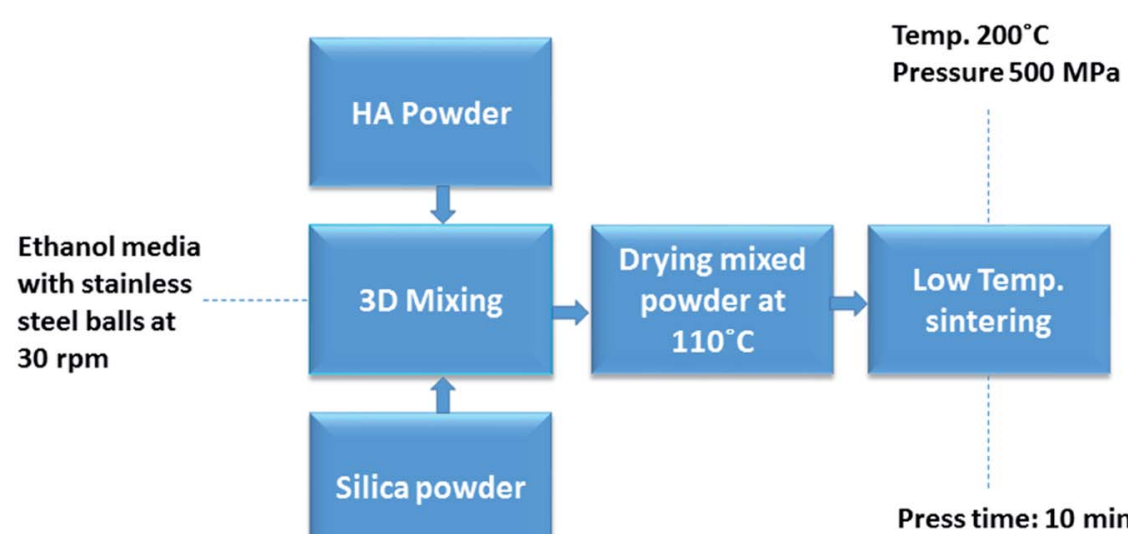

Fig. 2 Schematic of pure Si-HAp and ICPs-sorbed Si-HAp mixing, compaction, and sintering.

a line passing through a center, and an average hardness value was determined for each pellet.

The bulk density of the samples was measured by Archimedes' method. Sintered pellets were immersed in boiling deionized water for $10 \mathrm{~min}$ prior to density measurement to remove trapped air. The percentage relative sintered density ( $\%$ $\mathrm{RD})$ was evaluated using eqn (1). ${ }^{40}$

$$
\% \mathrm{RD}=\frac{\rho_{\mathrm{b}}}{\rho_{\mathrm{R}}} \times 100
$$

where $\rho_{\mathrm{b}}$ is the bulk density of the sintered sample measured by Archimedes' method and $\rho_{\mathrm{R}}$ is the theoretical density of the samples. The theoretical density of the samples was normalized and the density values of $2.94 \mathrm{~g} \mathrm{~cm}^{-3}$ and $2.2 \mathrm{~g} \mathrm{~cm}^{-3}$ were used for $\mathrm{HAp}^{3}$ and $\mathrm{SiO}_{2},{ }^{41}$ respectively.

Inductively coupled plasma-optical emission spectroscopy (ICP-OES) was used to determine the concentration of various elements in aqueous solution and sintered samples. The leachate collected during the durability test was also analyzed for the leached quantities of the matrix elements.

Chemical stability is an important parameter to ensure the durability and leach resistance of the developed waste matrix. Different dynamic and static tests developed by the American Society of Testing Materials (ASTM) and Material Characterization Centre (MCC) to investigate the matrix durability in aqueous solutions. In this case, the product consistency test (PCT) devised by ASTM C1285-02 was used under static conditions to measure the normalized leach rates of the constituent elements of the sintered matrix. ${ }^{33,42}$

\section{Results and discussions}

XRD patterns were measured to investigate the phase purity and crystal structure of pure HAp sintered samples and silica incorporated HAp sintered samples (Fig. 3). The broad peaks show low crystallinity and amorphous nature of the dried HAp and Si-HAp. It is also known from Fig. 3 that the peaks in pure HAp are identical to those in Si-HAp, suggesting the integrity of the apatite structure even with an increase in the silica dose up to $25 \mathrm{wt} \%$. Moreover, due to the amorphous nature of silica,
XRD peaks only reflect the presence of the pure HAp phase. The phase purity of HAp and HAp containing different silica compositions was confirmed by comparing the XRD peaks with JCPDS PDF No. 09-432. ${ }^{43}$

FTIR spectra shown in Fig. 4 confirmed the incorporation of silica into the HAp matrix in the range of 1 to $25 \mathrm{wt} \%$. For comparison, two samples were analyzed with $0 \mathrm{wt} \%$ silica and $100 \mathrm{wt} \%$ silica. The characteristic absorption peaks of the phosphate group $\left(\mathrm{PO}_{4}{ }^{2-}\right)$ appear at 566, 603, 963 and $1044 \mathrm{~cm}^{-1}$. A small peak around $875 \mathrm{~cm}^{-1}$ (in all samples except

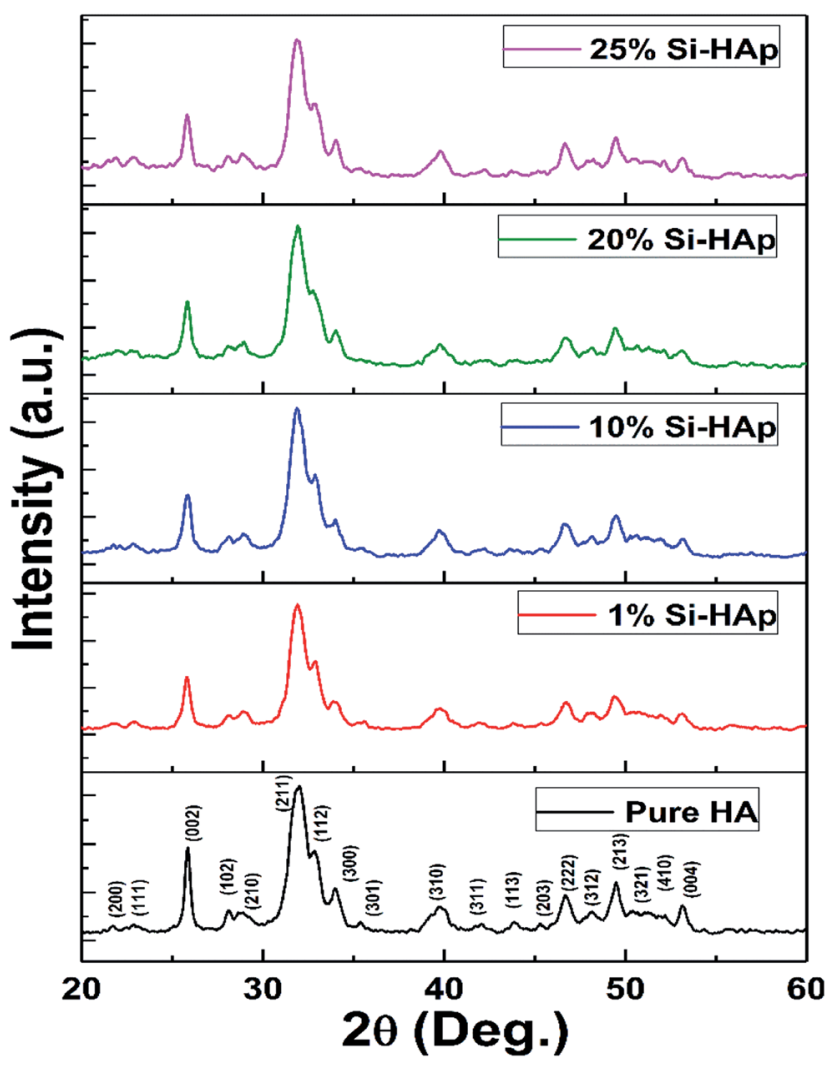

Fig. 3 XRD patterns of the nano-crystalline Si-HAp composite sintered at $200^{\circ} \mathrm{C}, 500 \mathrm{MPa}, 10 \mathrm{~min}$ in comparison to the pure dried HAp. 


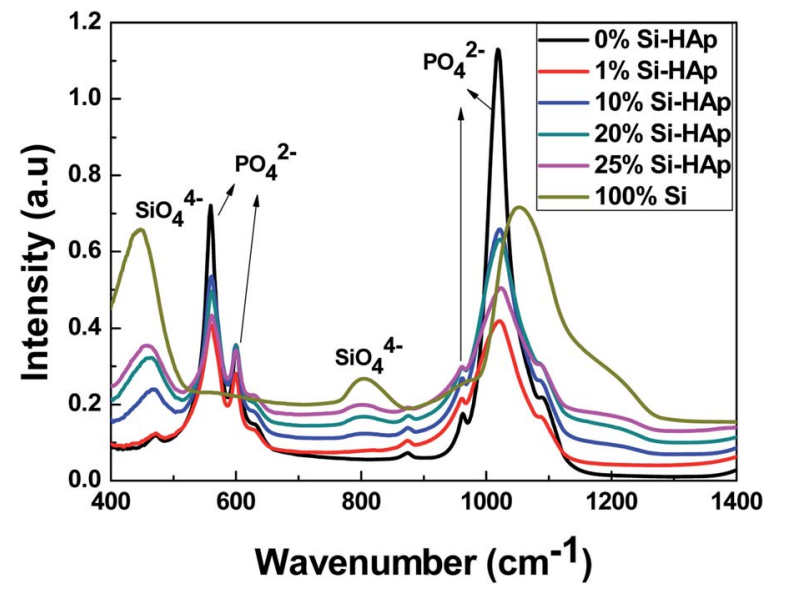

Fig. 4 FTIR spectra of Si-HAp sintered composite in comparison with pure Si and HAp.

pure silica) can be attributed to carbonates. Carbonates can be substituted by phosphates due to the absorption of $\mathrm{CO}_{2}$ from the environment under alkaline conditions $(\mathrm{pH}=10.3)$ during synthesis. ${ }^{44}$ The appearance of two new peaks at the wavenumbers 444 and $814 \mathrm{~cm}^{-1}$ was assigned to the $\mathrm{SiO}_{4}{ }^{4-}$ groups, clearly demonstrating the presence of silica in the HAp matrix ${ }^{43,45}$ as the peak intensity increased with raising silica incorporation. Gibson et al. reported the incorporation of silica at up to $0.4 \mathrm{wt} \%$ into apatite synthesized by wet precipitation reaction method. ${ }^{46}$ They proposed a replacement of the phosphate group $\left(\mathrm{PO}_{4}{ }^{2-}\right)$ with the silicate $\left(\mathrm{SiO}_{4}{ }^{4-}\right)$ group during the synthesis process, which is different from our solid-state mixing of separately synthesized silica and HAp components. Bogya et al. reported the existence of non-incorporated amounts of silica in the form of a silica polymer coating on the nano-grains of HAp. ${ }^{22}$

During sintering, the additional amount of silica nanoparticles can diffuse at the grain boundaries to form a glassy layer, resulting in densification and good mechanical properties. The silica nanoparticles possess a high surface energy value due to the very small particle size up to $7 \mathrm{~nm}$ and the large surface area $\left(495 \mathrm{~m}^{2} \mathrm{~g}^{-1}\right)$ compared to synthesized HAp $\left(112 \mathrm{~m}^{2}\right.$ $\mathrm{g}^{-1}$ ). These properties also suggest that it is a better candidate for micro-encapsulation when it is in excess. Thus, the good physico-mechanical properties of the consolidated matrix may be due to the silica incorporated HAp structure and microencapsulation phenomena.

Fig. 5 shows the fractured surface micrographs of sintered samples of pure HAp and Si-HAp with different wt\% of silica. The cold sintering has caused a limited grain growth, and the sintered pure matrix of HAp was composed of nano-grains. ${ }^{40}$ Silica itself is known as a grain growth inhibitor and produces sintered bodies with limited or very small grain growth.$^{47}$ Low temperature sintered Si-HAp composites have shown a homogeneous microstructure in this study due to good 3D mixing technique (Fig. 5). ${ }^{22}$ The presence of silica might have contributed to achieve good densification. This effect may be attributed to the accumulation and consolidation of $\mathrm{SiO}_{2}$ along with the amorphous phases of HAp at the pores under the combined effect of cold sintering parameters (i.e. uniaxial pressure and temperature) (Fig. 5a-e). ${ }^{48}$

The EDX mapping of $25 \mathrm{wt} \%$ ICPs-Si and $75 \mathrm{wt} \%$ ICPs-HAp is shown in Fig. 5f-n. From the images, it is obvious that the sample is highly densified with the homogenized distribution of all the ICPs (Fe, Co, Ni, Cu, Cr, $\mathrm{Zn}$, and $\mathrm{Mn}$ ).

The effect of silica incorporation on the \% $\mathrm{RD}$ and microhardness of the sintered samples is shown in Fig. 6. The measured physico-mechanical properties of the cold sintered $25 \mathrm{wt} \%$ Si-HAp composite confirmed the successful sintering at $200{ }^{\circ} \mathrm{C}$ and $500 \mathrm{MPa}$ within $10 \mathrm{~min}$. Cold sintered samples (25 wt\% ICPs-Si and $75 \mathrm{wt} \%$ ICPs-HAp) showed a good microhardness of $2.9 \pm 0.3 \mathrm{GPa}$. The \% RD and micro-hardness results have shown an increasing trend with the increase in $\mathrm{wt} \%$ of silica. The increase in \% RD and hardness may be associated with the condensed packing of porous cavities in HAp by silica diffusion during cold sintering which is a pressure-driven thermo-chemical process. Similar effects of silica incorporation on mechanical properties of concrete have been observed elsewhere at different experimental conditions. ${ }^{48,49}$

Fig. 7 shows the stress-strain curve for the sintered sample of $25 \mathrm{wt} \%$ ICPs-Si and $75 \mathrm{wt} \%$ ICPs-HAp composite. From Fig. 7 , it is apparent that the consolidated matrix is strong enough to stand the compressive load of up to $358 \mathrm{MPa}$. The bending and compressive strengths of dense HAp ceramics are in the wide range of 40-250 MPa and 120-900 MPa, respectively. ${ }^{50-54}$ The variation in mechanical properties can be attributed to the statistical nature of the strength distribution, which is affected by the residual microporosity, grain size, impurities, synthesis and sintering route $e t c .{ }^{54}$

All results discussed in the above-mentioned studies support deformation tendency in HAp, and it behaves differently than the typical ceramics such as aluminum oxide and glass. This load bearing behavior of the HAp matrix can provide the extra mechanical stability of the waste form in the event of sudden mechanical shocks and accidents.

Radioactive waste can be lethal to human and hazardous to the environment and should be stored safely under the multilayered barrier. The embedded components of the matrix may undergo chemical degradation under extreme environmental conditions, which can cause a leakage of hazardous waste to the environment. Therefore, the standard PCT was performed to evaluate the normalized leach rates (NLRi) for $25 \mathrm{wt} \%$ ICPs-Si and $75 \mathrm{wt} \%$ ICPs-HAp composite for $\mathrm{Ca}, \mathrm{Co}, \mathrm{Cr}, \mathrm{Cu}, \mathrm{Fe}, \mathrm{Mn}$, $\mathrm{Ni}, \mathrm{P}, \mathrm{Si}$ and $\mathrm{Zn}$, that are presented in Table 1 .

The obtained NLRi values ranged from $5.9 \times 10^{-9}$ for Fe to $1.2 \times 10^{-5} \mathrm{~g}$ per $\mathrm{m}^{2}$ per day for $\mathrm{Si}$ revealed good chemical stability of sintered composite for hosted elements. The measured NLRi values for Ca and P were found in close agreement with the literature values. ${ }^{55,56}$ Hassan et al. studied the possibility of cobalt immobilization by pure HAp under the identical sintering condition to our study. ${ }^{33}$ Their reported NLRi value for cobalt $\left(2.5 \times 10^{-5} \mathrm{~g}\right.$ per $\mathrm{m}^{2}$ per day $)$ is very close to our reported value for cobalt $\left(3.3 \times 10^{-5} \mathrm{~g}\right.$ per m${ }^{2}$ per day). However, the NLRi values for $\mathrm{Ca}\left(1.5 \times 10^{-5} \mathrm{~g} \mathrm{per}^{2}\right.$ per day $)$ and $\mathrm{P}(3.2 \times$ $10^{-5} \mathrm{~g}$ per $\mathrm{m}^{2}$ per day) are higher as compared to our reported 

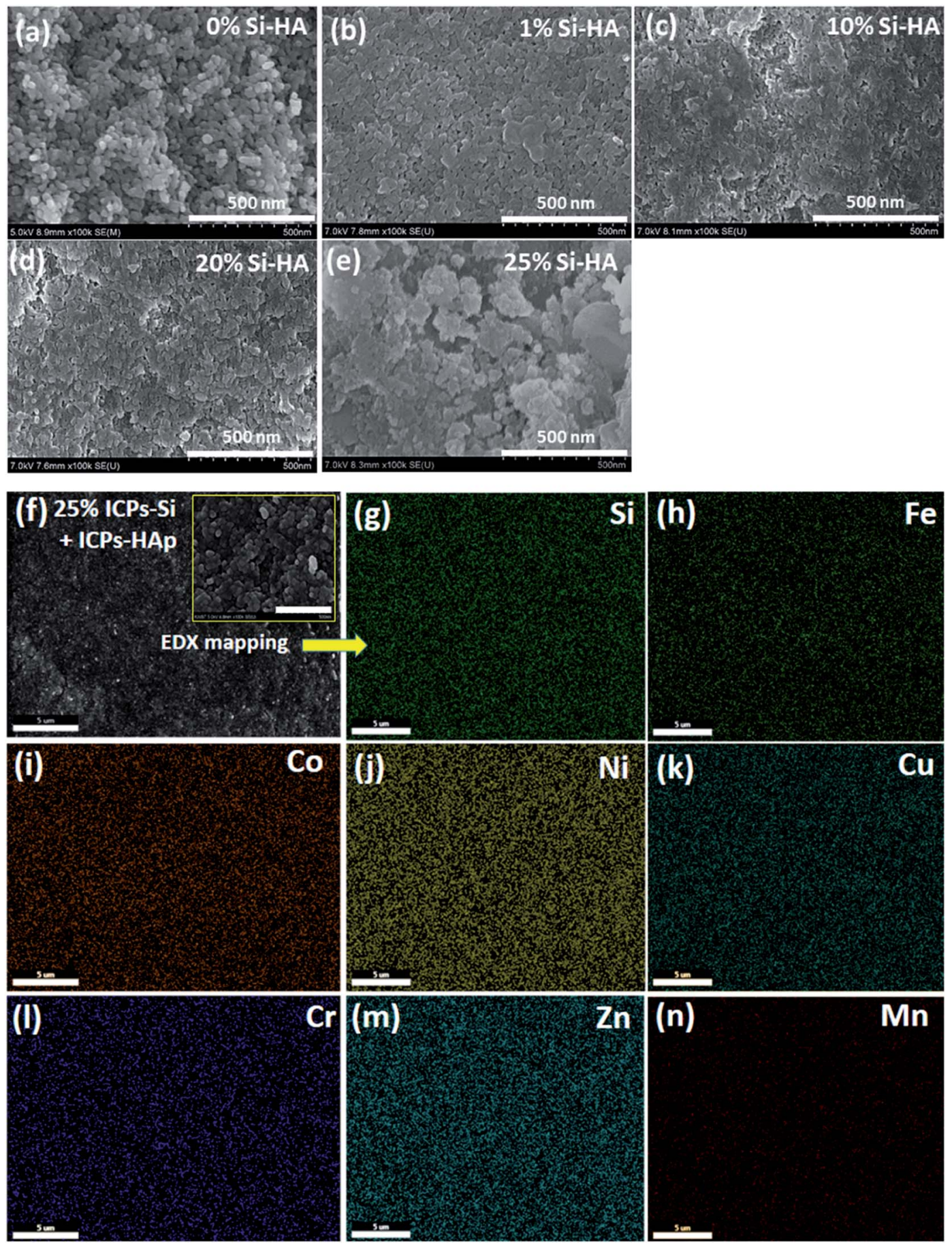

Fig. 5 (a-e) SEM images of pure and silica mixed HAp; (f) EDX elemental mapping showing the distribution and increased amount of silica; images $(\mathrm{g}-\mathrm{n})$ show the EDX mapping of $25 \mathrm{wt} \%$ ICPs-Si and $75 \mathrm{wt} \%$ ICPs-HAp.

composite values. The presence of silica in HAp might have posed an extra barrier by making a silicate-rich layer at a higher sintered density to retain different ions, especially $\mathrm{Ca}$ and $\mathrm{P}{ }^{45,57}$ It is hard to make a direct comparison with the limited experimental results in the literature, which were achieved at different compositions, sintering and testing conditions. However, the reported dissolution behavior of iodate substituted HAp, Co-HAp and our measured values are in close agreement to qualify the stability performance.
Based on our previous studies ${ }^{33,58}$ and current results, the reported immobilization route may be used for the solidification of radionuclides by capturing them from the liquid and gaseous wastes. The developed matrix might be used to immobilize gaseous waste (radioiodine) captured by fumed silica, silver functionalized silica or bismuth functionalized silica based gas adsorbents/filters. It is worth mentioning that the reported sintering temperature is very low and is safe for the immobilization of volatile radionuclides such as radioiodine. ${ }^{58}$ 


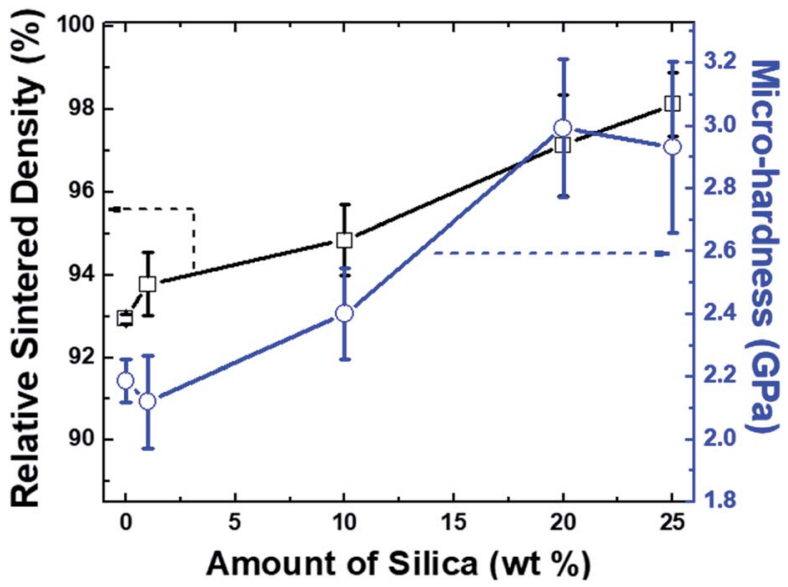

Fig. 6 Effect of silica contents on the relative sintered density (black) and micro-hardness (blue) of HAp; error bars can also be seen at each data point.

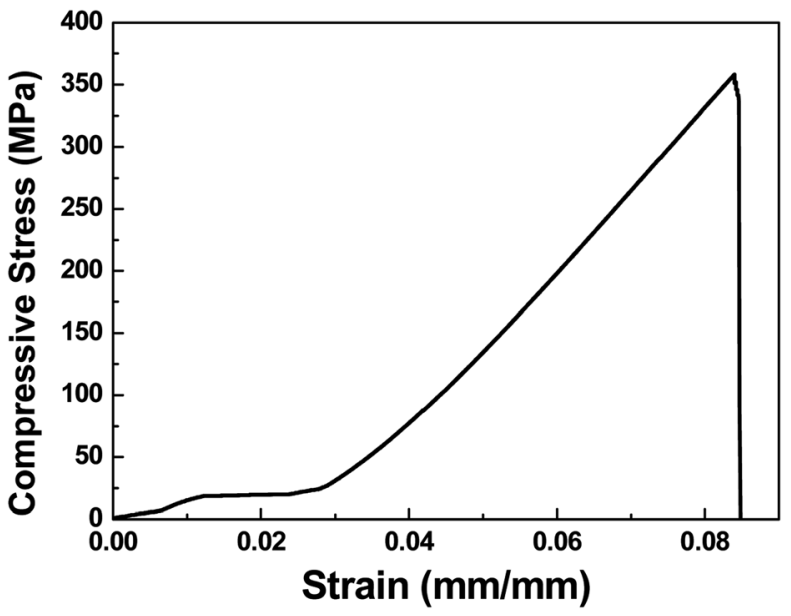

Fig. 7 The stress vs. strain curve of the composite with 25 wt\% ICPs-Si and 75 wt\% ICPs-HAp. The cross head speed was maintained at 0.1 $\mathrm{mm} \mathrm{min}^{-1}$ and the load was applied at a cross-section along the $z$ direction of the cylindrical pellet. The length-to-diameter ratio of the pellets was almost equal to 1 .

Table 1 Normalized leach rate ( $\mathrm{g}$ per $\mathrm{m}^{2}$ per day) for a waste composite with $25 \mathrm{wt} \%$ ICPs-Si and $75 \mathrm{wt} \%$ ICPs-HAp

\begin{tabular}{ll}
\hline Element & NLRi $\left(\mathrm{g}\right.$ per $\mathrm{m}^{2}$ per day) \\
\hline $\mathrm{Ca}$ & $5.3 \times 10^{-7}$ \\
$\mathrm{Co}$ & $3.3 \times 10^{-5}$ \\
$\mathrm{Cr}$ & $2.0 \times 10^{-6}$ \\
$\mathrm{Cu}$ & $1.9 \times 10^{-5}$ \\
$\mathrm{Fe}$ & $5.9 \times 10^{-9}$ \\
$\mathrm{Mn}$ & $1.4 \times 10^{-8}$ \\
$\mathrm{Ni}$ & $2.3 \times 10^{-5}$ \\
$\mathrm{P}$ & $3.1 \times 10^{-7}$ \\
$\mathrm{Si}$ & $1.2 \times 10^{-5}$ \\
$\mathrm{Zn}$ & $5.1 \times 10^{-7}$
\end{tabular}

\section{Conclusions}

In this paper, we investigated the possible use of waste to accommodate another waste with a view of reducing secondary waste volume. The sintering conditions for the Si-HAp composite have been successfully optimized to a loading of $25 \mathrm{wt} \%$ silica. The composite matrix provided excellent microhardness $(2.9 \pm 0.3 \mathrm{GPa})$ with a relative sintered density of $98.0 \pm 1.3 \%$ after cold sintering at $200{ }^{\circ} \mathrm{C}$. The waste matrix revealed excellent structure integrity by showing the compressive strength of $358 \mathrm{MPa}$. The cold sintering route for immobilization of waste containing $25 \mathrm{wt} \%$ ICPs-Si and $75 \mathrm{wt} \%$ ICPsHAp was successfully demonstrated. The durability of the cold sintered composite matrix showed a promising low leach rates $\left(5.9 \times 10^{-9}\right.$ to $1.2 \times 10^{-5} \mathrm{~g}$ per $\mathrm{m}^{2}$ per day) for all matrix elements. Low temperature, fast process time and sintering methods without artificial binders/additive-free make this immobilization route for radioactive waste an environmentally friendly, energy-efficient and simplified process.

\section{Conflicts of interest}

There are no conflicts to declare.

\section{Acknowledgements}

This research was supported by Basic Science Research Program through the National Research Foundation of Korea (NRF) funded by the Ministry of Science, ICT \& Future Planning (NRF2016R1A5A1013919).

\section{References}

1 IAEA-TECDOC-408, Application of Ion Exchange Processes for the Treatment of Radioactive Waste and Management of Spent Ion Exchangers, 2002.

2 IAEA-TECDOC-1022, New methods and techniques for decontamination in maintenance or decommissioning operations, 1998.

3 S. Iqbal, M. ul Hassan, H. J. Ryu and J.-I. Yun, J. Nucl. Mater., 2018, 507, 218-225.

4 N. H. Hamodi, K. A. Papadopoulou, T. Lowe and T. Abram, New J. Glass Ceram., 2012, 2, 111-120.

5 IAEA-RDS-2/38, Nuclear Power Reactors in the World, 2018.

6 L. R. Van Loon and W. Hummel, Nucl. Technol., 1999, 128, 388-401.

7 IAEA, Treatment of Spent Ion-Exchange Resins for Storage and Disposal, STI/DOC/lO/254, 1985.

8 IAEA-TECDOC-1051, Management of radioactive waste from ${ }^{99}$ Mo production, 1998.

9 R. O. A. Rahman, R. Z. Rakhimov, N. R. Rakhimova and M. I. Ojovan, Cementitious Materials for Nuclear Waste Immobilization, John Wiley \& Sons, UK, 2015.

10 J. H. Song, J. Environ. Manage., 2018, 206, 846-852.

11 G. Steinhauser, A. Brandl and T. E. Johnson, Sci. Total Environ., 2014, 470-471, 800-817.

12 G. Yang, H. Tazoe and M. Yamada, Sci. Rep., 2016, 6, 1-8. 
13 G. Kirchner, P. Bossew and M. De Cort, J. Environ. Radioact., 2012, 114, 35-40.

14 M. I. Ojovan and W. E. Lee, An Introduction to Nuclear Waste Immobilisation, Elsevier B.V, Sheffield, 2005.

15 M. I. Ojovan, G. A. Varlackova, Z. I. Golubeva and O. N. Burlaka, J. Hazard. Mater., 2011, 187, 296-302.

16 J. Kotátková, J. Zatloukal, P. Reiterman and K. Kolář, J. Environ. Radioact., 2017, 178-179, 147-155.

17 IAEA-TECDOC-972, Technologies for in situ immobilization and isolation of radioactive wastes at disposal and contaminated sites, 1987.

18 G. Li, Z. Zhao, J. Liu and G. Jiang, J. Hazard. Mater., 2011, 192, 277-283.

19 D. Q. Melo, V. O. S. Neto, J. T. Oliveira, A. L. Barros, E. C. C. Gomes, G. S. C. Raulino, E. Longuinotti and R. F. Nascimento, J. Chem. Eng. Data, 2013, 58, 798-806.

$20 \mathrm{~L}$. Wu, W. Forsling and P. W. Schindler, J. Colloid Interface Sci., 1991, 147, 178-185.

21 S. Iqbal and J.-I. Yun, Microporous Mesoporous Mater., 2017, 248, 149-157.

22 E. S. Bogya, R. Barab, A. Csvadari, L. Bizo and V. R. Dejeu, Chem. Pap., 2009, 63, 568-573.

23 M. J. Robles-Águila, J. A. Reyes-Avendaño and M. E. Mendoza, Ceram. Int., 2017, 43, 12705-12709.

24 J. C. Elliot, in Studies in Inorganic Chemistry, Elsevier Science B.V., 1989, vol. 18, p. 371.

25 G. C. Huang and J. K. Lee, Composites, Part A, 2010, 41, 473479.

26 V. Campana, G. Milano, E. Pagano, M. Barba, C. Cicione, G. Salonna, W. Lattanzi and G. Logroscino, J. Mater. Sci.: Mater. Med., 2014, 25, 2445-2461.

27 S. Iqbal and J.-I. Yun, RSC Adv., 2018, 8, 32211-32220.

28 L. Borum and O. C. Wilson, Biomaterials, 2003, 24, 36813688.

29 X. W. Li, H. Y. Yasuda and Y. Umakoshi, J. Mater. Sci.: Mater. Med., 2006, 17, 573-581.

30 J. L. Xu and K. A. Khor, J. Inorg. Biochem., 2007, 101, 187-195.

31 M. Aminzare, A. Eskandari, M. H. Baroonian, A. Berenov, Z. Razavi Hesabi, M. Taheri and S. K. Sadrnezhaad, Ceram. Int., 2013, 39, 2197-2206.

32 J. Wang, Front. Earth Sci., 2015, 3, 1-11.

33 M. ul Hassan, S. Iqbal, J.-I. Yun and H. J. Ryu, J. Hazard. Mater., 2019, 374, 228-237.

34 J. Guo, H. Guo, A. L. Baker, M. T. Lanagan, E. R. Kupp, G. L. Messing and C. A. Randall, Angew. Chem., Int. Ed., 2016, 55, 11457-11461.

35 J. Guo, A. L. Baker, H. Guo, M. Lanagan and C. A. Randall, J. Am. Ceram. Soc., 2017, 100, 669-677.

36 J. Wang and L. L. Shaw, Adv. Mater., 2007, 19, 2364-2369.
37 L. Campayo, A. Grandjean, A. Coulon, R. Delorme, D. Vantelon and D. Laurencin, J. Mater. Chem., 2011, 21, 17609-17611.

38 S. Handley-Sidhu, J. A. Hriljac, M. O. Cuthbert, J. C. Renshaw, R. A. D. Pattrick, J. M. Charnock, B. Stolpe, J. R. Lead, S. Baker and L. E. Macaskie, Environ. Sci. Technol., 2014, 48, 6891-6898.

39 M. ul Hassan and H. J. Ryu, in 42nd International Conference $\&$ Exposition on Advanced Ceramics and Composites, ed. The American Ceramic Society, Daytona Beach, Florida, 2018, pp. 123-124.

40 J. Wang and L. L. Shaw, Biomaterials, 2009, 30, 6565-6572.

41 J. Liu, G. Zong, L. He, Y. Zhang, C. Liu and L. Wang, Micromachines, 2015, 6, 855-864.

42 ASTM C1285-02, Standard Test Methods for Determining Chemical Durability of Nuclear, Hazardous and Mixed Waste Glasses and Multiphase Glass Ceramics: The Product Consistency Test (PCT) 1, ASTM, Conshohocken, USA, 2002, vol. 15, pp. 1-26.

43 T. Tian, D. Jiang, J. Zhang and Q. Lin, Mater. Sci. Eng., C, 2008, 28, 57-63.

44 C. W. Chen, C. S. Oakes, K. Byrappa, R. E. Riman, K. Brown, K. S. TenHuisen and V. F. Janas, J. Mater. Chem., 2004, 14, 2425-2432.

45 I. R. Gibson, S. M. Best and W. Bonfield, J. Biomed. Mater. Res., 1999, 44, 422-428.

46 J. A. Gómez del Río, P. J. Morando and D. S. Cicerone, J. Environ. Manage., 2004, 71, 169-177.

47 A. Kocjan, M. Logar and Z. Shen, Sci. Rep., 2017, 7, 2541.

48 S. Shihada and M. Arafa, Asian J. Mater. Sci., 2010, 2, 137146.

49 Y. Xu and D. D. L. Chung, Cem. Concr. Res., 1999, 29, 451453.

50 J. C. Elliott, Structure and Chemistry of the Apatite and Other Calcium Orthophosphate, Elsevier B.V, 1994.

51 S. Pramanik, A. K. Agarwal, K. N. Rai and A. Garg, Ceram. Int., 2007, 33, 419-426.

52 A. E. Hannora and S. Ataya, J. Alloys Compd., 2016, 658, 222233.

53 R. H. Doremus, J. Mater. Sci., 1992, 27, 285-297.

54 W. Suchanek and M. Yoshimura, J. Mater. Res., 1998, 13, 94117.

55 A. Coulon, A. Grandjean, D. Laurencin, P. Jollivet, S. Rossignol and L. Campayo, J. Nucl. Mater., 2017, 484, 324-331.

56 Y. H. Hsieh, S. A. Humphry-Baker, D. Horlait, D. J. Gregg,

E. R. Vance and W. E. Lee, J. Nucl. Mater., 2018, 509, 43-53.

57 L. L. Hench, J. Am. Ceram. Soc., 1991, 74, 1487-1510.

58 M. ul Hassan and H. J. Ryu, J. Nucl. Mater., 2019, 514, 84-89. 